\title{
Prospective Study of Early MRI Appearances following Flow- Diverting Stent Placement for Intracranial Aneurysms
}

\author{
B.J. McGuinness, S. Memon, and J.K. Hope
}

\begin{abstract}
BACKGROUND AND PURPOSE: MR imaging findings of aneurysm enlargement, aneurysm wall enhancement, perianeurysmal edema, and embolic phenomena following deployment of flow-diverting stents may be relevant to those patients who subsequently experience procedure-related intracranial hemorrhage. We sought to document the routine early postoperative MR imaging findings following flow-diverting stent insertion.
\end{abstract}

MATERIALS AND METHODS: Patients requiring flow-diverting stent placement for treatment or retreatment of cerebral aneurysms were prospectively included in the study during a 26-month period. Early postprocedural MR imaging studies were obtained and compared with preoperative imaging. Patient clinical outcome data were also collected.

RESULTS: There were 34 stent-placement procedures during the study period. Aneurysm mural enhancement and mild new perianeurysmal edema were present in 50\% and 14\%, respectively. Any DWI lesion was present in $57 \%$ of cases. New or possibly new foci of susceptibility effect were found ipsilateral to the stent and not associated with diffusion restriction in $66 \%$ of cases. There were 2 cases (6\%) of parenchymal hemorrhage and 2 major clinical complications (6\%) causing permanent morbidity.

CONCLUSIONS: Asymptomatic aneurysm mural enhancement is frequently seen following flow-diverting stent placement and should not necessarily be interpreted as a sign of impending aneurysm rupture. This finding often persists despite complete aneurysm occlusion. New small brain parenchymal susceptibility foci following stent placement have not previously been reported, to our knowledge, but were common in our series.

$\mathbf{F}$

ollowing flow-diverting stent placement to treat intracranial artery aneurysms, complications of delayed aneurysmal and parenchymal hemorrhage, with or without preceding symptomatology, have been described. ${ }^{1-5}$ Speculation remains regarding the etiology of both forms of hemorrhage, with an overall hemorrhage risk of $7 \%$ in reported series. ${ }^{6}$ Delayed parenchymal hemorrhages typically occur on the side of the treated aneurysm and at an incidence in excess of that expected due to dual antiplatelet medication alone and from that reported in prior non-flow-diverting-stent literature. ${ }^{7,8}$ MR imaging case reports have identified perianeurysmal edema, aneurysm expansion, and aneurysm wall enhancement in symptomatic patients before delayed aneurysm rupture. ${ }^{1,2}$ It is unknown whether such findings are predic-

Received August 11, 2014; accepted after revision November 3.

From the Department of Radiology (B.J.M., J.K.H.), Auckland City Hospital, Auckland, New Zealand; and Department of Radiology (S.M.), Vancouver General Hospital, Vancouver, British Columbia, Canada.

Please address correspondence to Ben McGuinness, MD, FRANZCR, Radiology Department, Auckland City Hospital, Park Rd, Grafton, Auckland, New Zealand; e-mail: benmcg@gmail.com

http://dx.doi.org/10.3174/ajnr.A4335

tive of subsequent aneurysm rupture. Microembolic change on diffusion imaging is common following angiographic procedures, and hemorrhagic transformation of infarcts is one putative cause of the parenchymal hemorrhages. We sought to document the routine early postprocedural MR imaging findings following flow-diverting stent insertion.

\section{MATERIALS AND METHODS \\ Study Population}

Local institutional ethics approval was obtained for this study. Consecutive patients requiring elective flow-diverting stent placement for the treatment of unruptured cerebral aneurysms or retreatment of previously ruptured aneurysms were prospectively included in the study during a 26-month period from January 2012 to March 2014. Acutely ruptured aneurysms were excluded.

\section{Stent-Placement Procedure}

Dual antiplatelet medication (300 mg aspirin and $75 \mathrm{mg}$ clopidogrel) was started 5 days before the intervention; then, clopidogrel was continued for 6 months, and aspirin, life-long. All procedures were performed with the patient under general anes- 
thesia. A 5000-U heparin bolus was given after femoral puncture; then, smaller boluses were given to keep activated clotting time at 2-3 times the baseline level. Postprocedural heparin was not routinely administered. Stents deployed were the Pipeline Embolization Device (Covidien, Irvine, California) in all cases except for 2 , in which the Flow-Redirection Endoluminal Device (MicroVention, Tustin, California) was used. Each stent-placement procedure was performed by 2 of 4 available interventional neuroradiologists with between 7 and 20 years of experience (ie, 2 operators per case).

\section{Postprocedural MR Imaging}

Early postprocedural MR imaging studies were planned between 2 and 10 days post-stent placement. All MR imaging scans were obtained on a 1.5T unit (Magnetom Avanto; Siemens, Erlangen, Germany). Scan protocol was sagittal and axial FSE T1, axial and coronal FSE T2, axial FLAIR, axial DWI, and either gradient-echo $\mathrm{T}^{*}$ or SWI also in the axial plane. TOF and dynamic contrastenhanced MRA (20 mL of gadopentetate dimeglumine, Magnevist; Bayer HealthCare Pharmaceuticals, Wayne, New Jersey) was performed followed by postcontrast axial FSE.

\section{Image Analysis}

Images from the early postprocedural MR imaging were interpreted by an experienced neuroradiologist (B.J.M.) and compared with any available prestenting imaging. Doubtful findings were resolved by consensus read by a second neuroradiologist (J.K.H.). Aneurysm size, shape, surrounding edema, wall enhancement, and the occurrence of parenchymal DWI-positive lesions or any other signal disturbance were recorded. Aneurysm size was considered stable if the maximum single dimension obtained was the same as or within $1 \mathrm{~mm}$ of that on the preprocedural imaging. Perianeurysmal edema was assessed on T2 and FLAIR and was defined as mild if $<3-\mathrm{mm}$ maximum thickness, moderate if between 3- and 10-mm thickness, and severe if $>10$ $\mathrm{mm}$. Aneurysm wall enhancement was defined as a complete rim of enhancement about the aneurysm wall. Pre-existing T1 shortening due to subacute thrombus or coil artifacts was excluded by reviewing the precontrast T1 scans. Enhancement due to intraaneurysmal flow was excluded by assessment of the TOF and contrast-enhanced MRA source images. Nodular enhancement was defined as mural enhancing foci of $>2 \mathrm{~mm}$ thick interspersed with thinner linear mural enhancement.

\section{Clinical and Delayed Follow-Up}

Clinical data were prospectively recorded as to neurologic symptomatology and clinical complications, at both the time of the intervention and at follow-up scans. All patients underwent imaging (typically MR imaging but occasionally DSA or CTA at the discretion of the attending interventional neuroradiologist) and clinical follow-up as per our standard protocol, which is 6 months, 1 year, and 2 years following stent placement.

\section{RESULTS}

\section{Study Population and Clinical Presentation}

Thirty-four elective flow-diverting stent procedures treating 33 aneurysms in 31 patients were performed during the study period (2 patients had a single aneurysm retreated with a flow-diverting stent during the study period, 1 patient had 2 aneurysms on 1 carotid segment treated with a single stent, and 1 patient had a bilateral paraclinoid aneurysms treated sequentially in 2 procedures). Twenty-seven patients were women, and 4, men, with an average age of 56 years (range, $42-81$ years). Median aneurysm pretreatment diameter was $12 \mathrm{~mm}$ (mean, $14 \mathrm{~mm}$; range, 5-35 $\mathrm{mm}$ ). All patients underwent clinical and imaging follow-up between 6 and 24 months. Eight cases were retreatment of previously coiled ruptured aneurysms, and the remainder were for unruptured aneurysms. Overall, 13 patients had coils present in the aneurysm, either deployed at the time of flow diversion or present from a previous coiling procedure. A single flow-diverting stent was used in all cases except for 5 in which between 2 and 4 stents were deployed. Four of the 26 unruptured aneurysms were symptomatic ( 2 unilateral headache, 1 headache and sixth cranial nerve palsy, 1 fourth cranial nerve palsy). Three aneurysms were in the vertebrobasilar territory with the remainder arising from the carotid artery ( 3 posterior communicating aneurysms). Four aneurysms were cavernous carotid with the remainder intradural in location.

Complete early postprocedural MR imaging studies were performed in 24 cases (21 patients and 22 aneurysms). Ten patients did not meet study imaging inclusion criteria and are excluded from MR imaging analysis: Four patients did not have MR imaging due to claustrophobia $(n=2)$ and booking errors $(n=2), 4$ patients did not receive contrast during their scans, and 2 patients had their scans outside the DWI target window (both at day 16). All patients underwent clinical and delayed imaging follow-up, and any patient who experienced clinical complications is discussed in the "Results" section, including any relevant findings from incomplete MR imaging performed.

\section{Early MR Imaging Findings}

Aneurysm mural enhancement was present in 11 of 22 (50\%) aneurysms measuring 1- to 3-mm thick. Three of these showed nodular wall enhancement, with the remainder being smooth and linear (Fig 1). Mild ( $\geq 3 \mathrm{~mm}$ ) perianeurysmal edema was present in 3 aneurysms (14\%, Fig 2). In all cases, prior imaging showed that this edema had developed secondary to flow-diverting stent insertion. Two of these patients re-presented to the hospital with headaches at days 5 and 23 postoperatively (another patient without perianeurysmal edema re-presented to the hospital with headache at day 21). Two of these patients with edema were retreated in this series in an attempt to promote aneurysm occlusion. None of the patients with perianeurysmal edema had coils within the treated aneurysm, while 6 of the 11 aneurysms (55\%) with mural enhancement had coils. Aneurysm size was stable or within $1 \mathrm{~mm}$ of pretreatment dimensions in all cases. Any DWI lesion was present in 13 of 24 cases (57\%). Seven cases (30\%) had $>5$ DWI lesions or any lesion $>5 \mathrm{~mm}$. Twenty-one of the 24 cases had susceptibility-weighted imaging performed, while 3 had gradient-echo T2. New foci of susceptibility effects ipsilateral to the stent and not associated with diffusion restriction were found in 8 patients (38\%) who had susceptibility-weighted sequences performed (Fig 3). A further $28 \%$ of these studies (6/21) had susceptibility foci ipsilateral to the stent, which were possibly new but did not have an earlier comparison SWI sequence. All SWI foci were $<2 \mathrm{~mm}$ and not identifiable on any other sequence. 


\section{Clinical Complications}

Ten clinical complications occurred in 9 of 34 operations (26\%). Eight of these were transient or resolved with appropriate treatment, and 2 patients (6\%) experienced permanent morbidity. One of these (parenchymal hemorrhage) was disabling ( $\mathrm{mRS}=$ 3 ), and the other (ischemic), nondisabling ( $\mathrm{mRS}=1)$. There were no deaths.

There were 3 ischemic and 2 hemorrhagic complications, 3 of 5 cases being transiently symptomatic. Four of these occurred within 48 hours of stent deployment, and 1 at 2 weeks (pontine hemorrhage). Two cases (6\%) of symptomatic parenchymal hemorrhage occurred within the treated vascular territory. One of these occurred at approximately 6 hours following stent deployment, resulting in a $4-\mathrm{cm}$ hematoma in the superior left temporal lobe with associated Sylvian subarachnoid hemorrhage and producing expressive and receptive dysphasia. This patient subsequently had MR imaging the following day, which showed 2 small foci of restricted diffusion $(<5 \mathrm{~mm})$ remote from the hemorrhage. No new foci of susceptibility effect were identified remote from the hemorrhage. This patient was excluded from the early MR imaging analysis due to not having contrast at the time of her acute scan. The

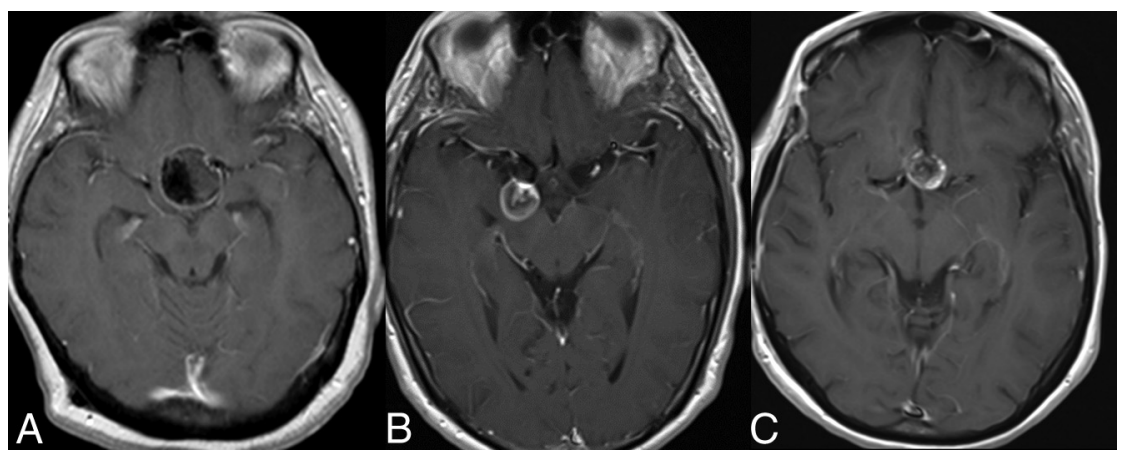

FIG 1. Postcontrast T1-weighted images showing aneurysm mural enhancement following flowdiverting stent placement. $A$, Smooth linear wall enhancement in a large left paraclinoid aneurysm. $B$, Linear wall enhancement along with some central aneurysm luminal filling in a large right posterior communicating artery aneurysm. $C$, Nodular wall enhancement of a left paraclinoid aneurysm.

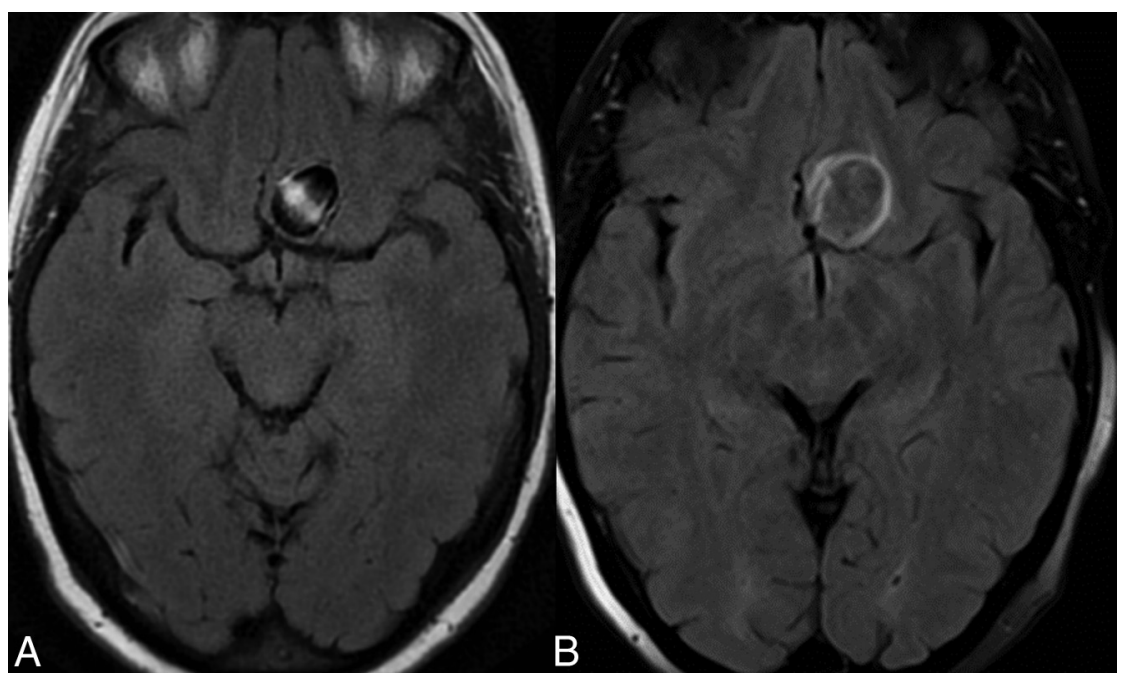

FIG 2. Development of mild perianeurysmal edema following flow-diverting stent placement. $A$, Axial FLAIR imaging before treatment. $B$, Corresponding FLAIR image following stent placement shows new perianeurysmal edema. second case of parenchymal hemorrhage was a 3-mm pontine hemorrhage found on CT at 2 weeks' post-stent deployment when the patient presented to another institution following a syncope episode (Fig 4). There were no lasting clinical sequelae, and no clear cause of the syncope was identified. No DWI or SWI focus was identified at the site of the hemorrhage on the patient's preceding early MR imaging scan at day 2. Both hemorrhages occurred in the vascular territory accessed by the guide catheter, the first case occurring distal to the stented cavernous carotid aneurysm and the second occurring proximal to a posterior cerebral artery aneurysm.

Three patients re-presented to the hospital with headaches without subsequent hemorrhage or neurologic sequelae. One of these patients re-presented to the hospital with headaches twice and was retreated with a flow-diverting stent within this series in an attempt to promote occlusion of the aneurysm. One patient, who also experienced a transient ischemic deficit, required open surgical repair of a femoral artery puncture site, with full recovery.

\section{Delayed Imaging Follow-Up}

All patients have had between 6- and 24-month follow-up. Imaging follow-up was with contrast-enhanced MRA in all patients except for 2 in whom CTA was performed. DSA was performed in addition to these studies in 5 cases. Aneurysm occlusion was complete in 19 of 33 aneurysms (58\%) and in 14 of $18(78 \%)$ that had at least 12 months of follow-up. Of the 11 aneurysms that showed aneurysm wall enhancement, 2 have resolved in association with aneurysm occlusion, 5 showed stable wall enhancement despite aneurysm occlusion, 3 showed stable wall enhancement in the presence of ongoing aneurysm filling, and 1 case with an occluded aneurysm was not assessable because it was followed with CTA. Of the 3 aneurysms with perianeurysmal edema, 1 resolved in association with aneurysm occlusion and reduction in aneurysm size, 1 is stable with persistent aneurysm filling, and 1 with an occluded aneurysm is not assessable due to CTA follow-up. The areas of new susceptibility foci on early post-stent-insertion MR imaging remain unchanged in size, number, and morphology.

\section{DISCUSSION}

Delayed intracranial hemorrhage is now a well-recognized risk following flow-diverting stent placement. However because the etiology of both forms of hemorrhage remain unclear, endovascular practitioners still have no reliable strategies to minimize this complication. In patients who re-present to the hospital with headache or other symptoms before potential delayed aneurysm rup- 
ture, there is the opportunity for therapeutic intervention such as modifying dual antiplatelet medications, inserting an additional flow-diverting stent, or performing parent artery occlusion. This study has shown that similar to results in the coiling literature, aneurysm mural enhancement is frequently seen on MR imaging in asymptomatic patients who do not proceed to aneurysm rupture. ${ }^{9}$ Aneurysm wall enhancement is thought to represent an inflammatory healing response and, when present following coiling, is usually seen to persist for months to years. ${ }^{9}$ Such an inflammatory response can be responsible for optic nerve or other cranial nerve dysfunction in the absence of aneurysm enlargement. Our series also shows that it can persist for

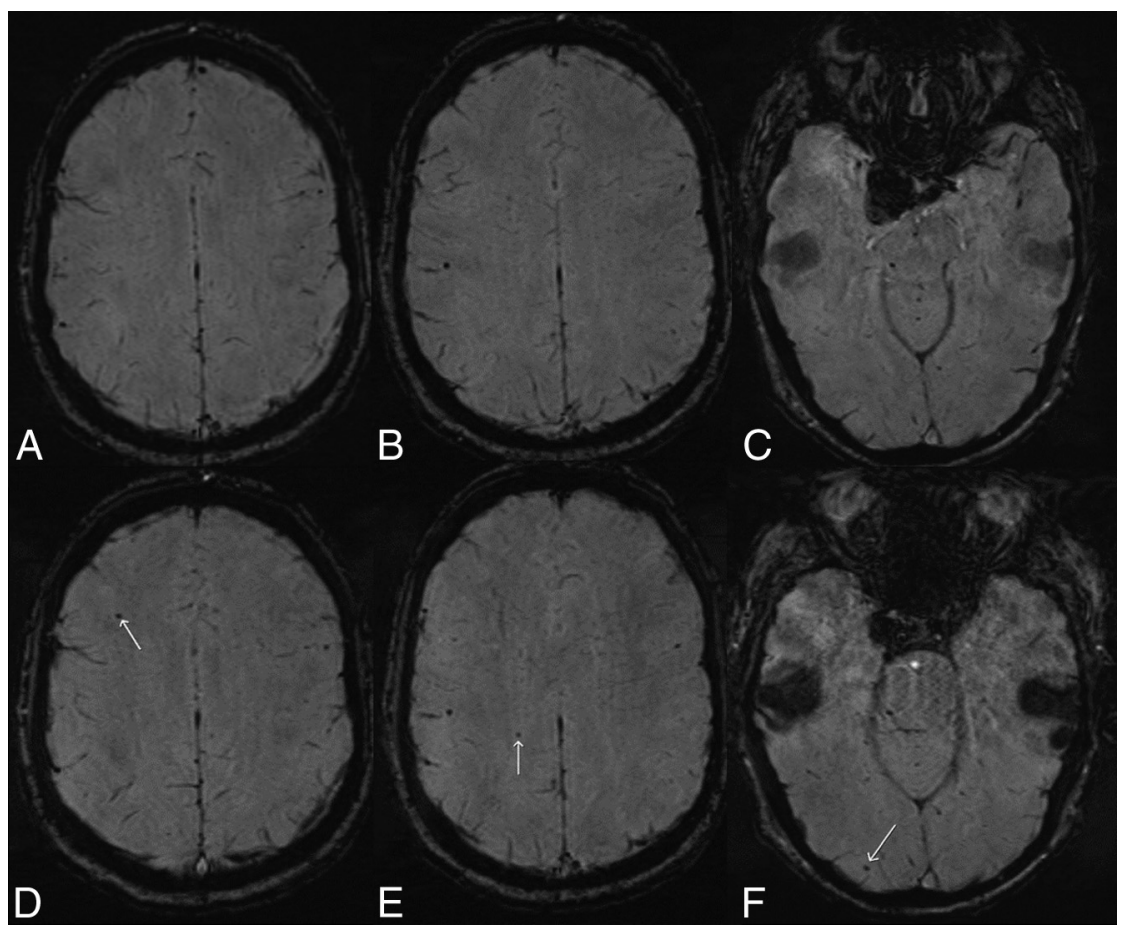

FIG 3. Development of new susceptibility foci in the ipsilateral hemisphere following flowdiverting stent placement for a right posterior communicating artery aneurysm. Axial SWI (top row, $A-C$ ) obtained 7 days before deployment of a right supraclinoid ICA flow-diverting stent shows a single susceptibility focus laterally in the superior right parietal lobe (B). Axial SWI obtained 6 days following stent deployment (bottom row, $D-F$ ) shows small new susceptibility foci in the superior right frontal lobe ( $D$, arrow), superior right parietal lobe (E, arrow) medial to the pre-existing focus, and in the right occipital pole $(F$, arrow). The patient had a moderate-sized posterior communicating artery arising from the aneurysm, approximately co-dominant in size with the right $\mathrm{Pl}$ segment. months to years following flow diversion despite complete aneurysm occlusion.

In the coiling literature, perianeurysmal edema has been observed before treatment in large and often partially thrombosed aneurysms but also in smaller aneurysms following coiling with both HydroCoils (MicroVention) and bare platinum coils. ${ }^{9,10}$ Our study showed that while perianeurysmal edema can occasionally occur following flow diversion, it is usually mild.

There were no cases of delayed aneurysm rupture in our series, and it is not possible to draw any conclusions about predictive findings from postprocedural MR imaging for delayed aneurysm rupture. Previous published case reports of delayed aneurysm rupture with MR imaging preceding rupture show findings ranging from stable aneurysm size and perianeurysmal edema but with linear wall enhancement to marked aneurysm enlargement and new brain edema. ${ }^{1,2} \mathrm{On}$ the basis of our findings and the previous published literature, aneurysm wall enhancement is common and should not be interpreted as a sign of impending rupture. Perianeurysmal edema is a less common finding and would be a more concerning finding, particularly in the symptomatic patient. Two patients in this series with perianeurysmal edema underwent further treatment, one of which resulted in successful aneurysm occlusion and resolution of the edema, while the other aneurysm continues to fill with stable edema.

Foci of restricted diffusion are known to be a common finding after aneurysm embolization and, to a lesser degree, diagnostic cerebral angiography. ${ }^{11,12}$ They are also increasingly recognized after open and endovascular cardiac interventions. ${ }^{13,14}$ The degree to which they are due to gas or thrombotic or other embolic material is unknown; however, the use of heparin and air filters has been shown to reduce their incidence. ${ }^{12}$

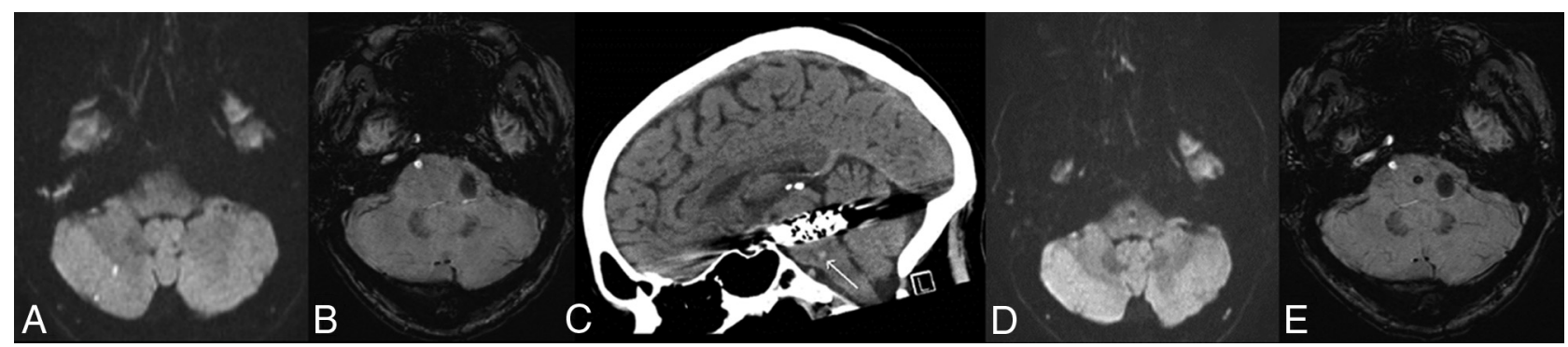

FIG 4. Pontine hemorrhage 2 weeks following flow-diverting stent placement for a large left posterior cerebral artery aneurysm in a 52 -year-old man. Axial DWI $(A)$ and SWI $(B)$ performed at 2 days post-stent deployment show 2 small foci of restricted diffusion in the right cerebellar hemisphere but no signal disturbance in the brain stem. Sagittally reformatted noncontrast CT scan (C) obtained 2 weeks after stent deployment following a syncopal episode shows a 3-mm pontine hemorrhage (arrow). Axial DWI (D) and SWI (E) performed at 6 months show the susceptibility effect at the site of the prior pontine hemorrhage. 
Diffusion-positive lesions are mostly asymptomatic, but larger or more numerous lesions are associated with ischemic symptoms. ${ }^{11}$ This was confirmed in our study with 2 of the 3 patients who had ischemic TIA or stroke with MR imaging scans showing a large number of diffusion lesions. The third patient did not have MR imaging, but an early CT scan and delayed MR imaging showed embolic changes that would certainly have been correlated with a large DWI load. Evidence for procedural techniques that minimize the incidence and number of DWI foci is lacking; such techniques will undoubtedly reduce the procedural risk of clinical stroke. Adverse long-term neuropsychological outcomes have been shown with high DWI load following cardiac surgery. Whether this translates to neuroendovascular treatments is unknown, and even if it does, it may still be of lower risk than open neurosurgical alternatives. ${ }^{15,16}$

There were 2 cases of parenchymal hemorrhage in our series. The first of these was reasonably typical relative to those previously reported in the literature. Proposed mechanisms include unrecognized microwire perforation, hemorrhagic transformation of an infarct, embolized foreign body material, hemodynamic changes secondary to the stent, and hyper-response to dual antiplatelet medication. ${ }^{6,17,18}$ Our second case of parenchymal hemorrhage was atypical, given the size, location, and lack of long-term neurologic morbidity. Most described parenchymal hemorrhages following flow diversion are supratentorial and result in a severe clinical deficit. ${ }^{3,5,17}$ Most interesting, in this case, we had the opportunity of obtaining MR imaging before the brain stem hemorrhage, but it did not show a focus of diffusion restriction or susceptibility effect as a clue to the cause of the hemorrhage. The only caveat was that DWI can fail to detect a small brain stem infarct when performed early after the stroke (day 2 postoperative in this case). ${ }^{19}$

New susceptibility foci, which almost always occurred distally within the vascular territory being treated with a flow-diverting stent, were an unexpected finding of this study. There was no correlation of these foci with areas of restricted diffusion. The case illustrated in this article (Fig 3) had an earlier comparison MR imaging that was only 14 days prior, meaning that incidental new microhemorrhages unrelated to the intervention are an implausible explanation. Potential causes include primary microhemorrhages, microhemorrhagic transformation of thromboembolism, gas embolism, or foreign body embolism. On making the observation of these new SWI foci, we reviewed our previous 20 elective non-stent-coiling cases and found the following: Three had definitely new SWI foci compared with a prior SWI study, 4 had probable new foci compared with a prior gradient-echo T2 study, 5 had possibly new foci with no old studies, and 8 had no SWI foci (B.J.M., unpublished data, March 2014). We suspect that this finding is not unique to flow-diverting stents.

\section{CONCLUSIONS}

Asymptomatic aneurysm mural enhancement is frequently seen following flow-diverting stent placement and should not necessarily be interpreted as a sign of impending aneurysm rupture. This finding often persists despite complete aneurysm occlusion. Perianeurysmal edema is less common and, particularly when symptomatic, is potentially an indication to perform further treatment. DWI data suggest a comparable embolic risk to that reported with aneurysm coiling. New small susceptibility foci following stent placement have not been previously reported, to our knowledge, but were common in our series.

Disclosures: Ben J. McGuinness_UNRELATED: Travel/Accommodations/Meeting Expenses Unrelated to Activities Listed: Covidien, Comments: I have attended Covidien-sponsored meetings where flights, accommodation, and meals have been paid. I have not received any direct payment from Covidien and have not been paid to attend these meetings. Sohrabh Memon-UNRELATED: Employment: University of British Columbia; Travel/Accommodations/Meeting Expenses Unrelated to Activities Listed: University of British Columbia Fellow conference stipend.

\section{REFERENCES}

1. Hampton T, Walsh D, Tolias C, et al. Mural destabilization after aneurysm treatment with a flow-diverting device: a report of two cases. J Neurointerv Surg 2011;3:167-71

2. Chow M, McDougall C, O'Kelly C, et al. Delayed spontaneous rupture of a posterior inferior cerebellar artery aneurysm following treatment with flow diversion: a clinicopathologic study. AJNR Am J Neuroradiol 2012;33:E46-51

3. Cruz JP, Chow M, O’Kelly C, et al. Delayed ipsilateral parenchymal hemorrhage following flow diversion for the treatment of anterior circulation aneurysms. AJNR Am J Neuroradiol 2012;33: 603-08

4. O’Kelly CJ, Spears J, Chow M, et al. Canadian experience with the Pipeline embolization device for repair of unruptured intracranial aneurysms. AJNR Am J Neuroradiol 2013;34:381-87

5. Tomas C, Benaissa A, Herbreteau D, et al. Delayed ipsilateral parenchymal hemorrhage following treatment of intracranial aneurysms with flow diverter. Neuroradiology 2014;56:155-61

6. Brinjikji W, Murad M, Lanzino G, et al. Endovascular treatment of intracranial aneurysms with flow diverters: a meta-analysis. Stroke 2013;44:442-47

7. Shapiro M, Becske T, Sahlein D, et al. Stent-supported aneurysm coiling: a literature survey of treatment and follow-up. AJNR Am J Neuroradiol 2012;33:159-63

8. Diener HC, Bogousslavsky J, Brass LM, et al. Aspirin and clopidogrel compared with clopidogrel alone after recent ischaemic stroke or transient ischaemic attack in high-risk patients (MATCH): randomised, double-blind, placebo-controlled trial. Lancet 2004;364:331-37

9. Fanning NF, Willinsky RA, ter Brugge KG. Wall enhancement, edema, and hydrocephalus after endovascular coil occlusion of intradural cerebral aneurysms. J Neurosurg 2008;108:1074-86

10. Craven I, Patel UJ, Gibson A, et al. Symptomatic perianeurysmal edema following bare platinum embolization of a small unruptured cerebral aneurysm. AJNR Am J Neuroradiol 2009;30: 1998-2000

11. Kang DH, Kim BM, Kim DJ, et al. MR-DWI-positive lesions and symptomatic ischemic complications after coiling of unruptured intracranial aneurysms. Stroke 2013;44:789-91

12. Bendszus M, Koltzenburg M, Bartsch AJ, et al. Heparin and air filters reduce embolic events caused by intra-arterial cerebral angiography: a prospective, randomized trial. Circulation 2004; 110:2210-15

13. Gaita F, Leclercq JF, Schumacher B, et al. Incidence of silent cerebral thromboembolic lesions after atrial fibrillation ablation may change according to technology used: comparison of irrigated radiofrequency, multipolar nonirrigated catheter and cryoballoon. J Cardiovasc Electrophysiol 2011;22:961-68

14. Floyd TF, Shah PN, Price CC, et al. Clinically silent cerebral ischemic events after cardiac surgery: their incidence, regional vascular occurrence, and procedural dependence. Ann Thorac Surg 2006;81: 2160-66

15. Barber PA, Hach S, Tippett LJ, et al. Cerebral ischemic lesions on 
diffusion-weighted imaging are associated with neurocognitive decline after cardiac surgery. Stroke 2008;39:1427-33

16. Scott RB, Eccles F, Molyneux AJ, et al. Improved cognitive outcomes with endovascular coiling of ruptured aneurysms: neuropsychological outcomes from the International Subarachnoid Aneurysm Trial (ISAT). Stroke 2010;41:1743-47

17. Deshmukh V, Hu YC, McDougall CG, et al. Histopathological assessment of delayed ipsilateral parenchymal hemorrhages after treatment of paraclinoid aneurysms with the Pipeline embolization device. Neurosurgery 2012;71:E551-52

18. Goh C, Churilov L, Mitchell P, et al. Clopidogrel hyper-response and bleeding risk in neurointerventional procedures. AJNR Am J Neuroradiol 2013;34:721-26

19. Fukuoka T, Takeda H, Dembo T, et al. Clinical review of $\mathbf{3 7}$ patients with medullary infarction. J Stroke Cerebrovasc Dis 2012; 21:594-99 